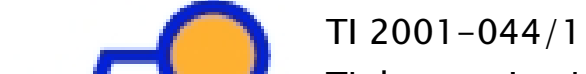 \\ Tinbergen Institute Discussion Paper \\ A Class of Consistent Share Functions for Games in \\ Coalition Structure
}

René van den Brink ${ }^{7}$

Gerard van der Laan'2,3

1 Department of Econometrics, Tilburg University,

2 Department of Econometrics, Faculty of Economics and Business Administration, Vrije Universiteit Amsterdam, 3 Tinbergen Institute 
Tinbergen Institute

The Tinbergen Institute is the institute for economic research of the Erasmus Universiteit Rotterdam, Universiteit van Amsterdam and

Vrije Universiteit Amsterdam.

Tinbergen I nstitute Amsterdam

Keizersgracht 482

1017 EG Amsterdam

The Netherlands

Tel.: +31.(0)20.5513500

Fax: $\quad+31 .(0) 20.5513555$

Tinbergen Institute Rotterdam

Burg. Oudlaan 50

3062 PA Rotterdam

The Netherlands

Tel.: $\quad+31 .(0) 10.4088900$

Fax: $\quad+31 .(0) 10.4089031$

Most TI discussion papers can be downloaded at

http://www.tinbergen.nl 


\title{
A Class of Consistent Share Functions for Games in Coalition Structure ${ }^{\dagger}$
}

\author{
René van den Brink* \\ Gerard van der Laan ${ }^{\ddagger}$
}

April 11, 2001

Correspondence to:

René van den Brink

Department of Econometrics

Tilburg University

P.O. Box 90153

5000 LE Tilburg

The Netherlands

\footnotetext{
†This research is part of the Research-program "Competition and Cooperation".

*Department of Econometrics and Operations Research, CentER, Tilburg University, P.O. Box 90153, Tilburg, The Netherlands. E-mail: jbrink@kub.nl. This author is financially supported by the Netherlands Organization for Scientific Research (NWO), ESR-grant 510-01-0504.

${ }^{\ddagger}$ Department of Econometrics and Tinbergen Institute, Vrije Universiteit, De Boelelaan 1105, Amsterdam, The Netherlands. E-mail: glaan@econ.vu.nl
} 


\begin{abstract}
A cooperative game with transferable utility -or simply a TU-game- describes a situation in which players can obtain certain payoffs by cooperation. A value function for these games is a function which assigns to every such a game a distribution of the payoffs over the players in the game. An alternative type of solutions are share functions which assign to every player in a TU-game its share in the payoffs to be distributed.

In this paper we consider cooperative games in which the players are organized into an a priori coalition structure being a finite partition of the set of players. We introduce a general method for defining a class of share functions for such games in coalition structure using a multiplication property that states that the share of player $i$ in the total payoff is equal to the share of player $i$ in some internal game within $i$ 's a priori coalition, multiplied by the share of this coalition in an external game between the a priori given coalitions. We show that these coalition structure share functions satisfy certain consistency properties. We provide axiomatizations of this class of coalition structure share functions using these consistency and multiplication properties.
\end{abstract}

JEL classification number: C71

Keywords: TU-Game, coalition structure, share function, multiplication property, consistency.

\title{
1 Introduction
}

A situation in which a finite set of $n$ players can obtain certain payoffs by cooperation can be described by a cooperative game with transferable utility -or simply a TUgame- being a pair $(N, v)$, where $N=\{1, \ldots, n\}$ is a finite set of players and $v: 2^{N} \rightarrow$ $\mathbf{R}$ is a characteristic function on $N$ such that $v(\emptyset)=0$. In this paper we consider monotone TU-games, i.e. games $(N, v)$ satisfying $v(E) \quad v(F)$ if $E \subset F \subset N$. We denote the collection of all monotone TU-games by $\mathcal{G}$. A monotone game $(N, v)$ is a null game if $v(E)=0$ for all $E \subset N$. We denote the null game on player set $N$ by $\left(N, v^{0}\right)$, and we denote the class of monotone games that are not null games by $\mathcal{G}^{+}$.

A value function on $\mathcal{G}$ is a function $f$ that assigns to every $(N, v) \in \mathcal{G}$ an $|N|$-dimensional real vector $f(N, v) \in \mathbf{R}^{|N|}$ representing a distribution of payoffs among the players. A value function $f$ is efficient on $\mathcal{G}$ if for every game in $\mathcal{G}$ it exactly distributes the worth $v(N)$ of the 'grand coalition' over all players, i.e. if $\sum_{i \in N} f_{i}(N, v)=v(N)$ for every $(N, v) \in \mathcal{G}$. An example of an efficient value function is the Shapley value (Shapley (1953)). An example of a value function that is 
not efficient is the Banzhaf value (Banzhaf (1965), Owen (1975), Dubey and Shapley (1979)). Since the Banzhaf value is not efficient it is not adequate in allocating the worth $v(N)$. In order to allocate $v(N)$ according to the Banzhaf value, van den Brink and van der Laan (1998) characterized the normalized Banzhaf value which distributes the worth $v(N)$ proportional to the Banzhaf values of the players.

An alternative approach to efficiently allocating the worth $v(N)$ is using share functions as introduced in van der Laan and van den Brink (1998). A share vector for game $(N, v) \in \mathcal{G}$ is an $|N|$-dimensional real vector $x \in \mathbf{R}^{|N|}$ such that $\sum_{i \in N} x_{i}=$ 1. Here $x_{i}$ is player $i$ 's share in the total payoff that is to be distributed among the players. A share function on $\mathcal{G}$ is a function that assigns to every $(N, v) \in \mathcal{G}$ exactly one share vector ${ }^{1} \rho(N, v) \in\left\{x \in \mathbf{R}^{|N|} \mid \sum_{i \in N} x_{i}=1\right\}$. The share function corresponding to the Shapley value is the Shapley share function which is obtained by dividing the Shapley value of each player by the sum of the Shapley values of all players (i.e. by $v(N)$ ). Similarly, the Banzhaf share function is obtained by dividing the Banzhaf- or normalized Banzhaf value by the corresponding sum of payoffs over all players. Note that, although the Banzhaf- and normalized Banzhaf value are very different (for example, the Banzhaf value satisfies linearity and the dummy player property which are not satisfied by the normalized Banzhaf value), they correspond to the same Banzhaf share function.

Share functions yield a distribution of the worth of the grand coalition reflecting the individual bargaining position of the players. In this paper we consider situations in which the grand coalition forms in order to maximize the total payoff, but in which the players are also organized into smaller coalitions. These coalitions form a coalition structure being a finite partition $P=\left\{P_{1}, \ldots, P_{m}\right\}$ of the player set $N$ and are assumed to be given exogenously. As motivated by Winter (1989), the coalitions can be seen as pressure groups for the division of $v(N)$. So, to divide the worth of the grand coalition over all players, first this worth is distributed over the coalitions in the a priori given coalition structure, and then the payoff assigned to a coalition is distributed over all its players.

For games in a priori given coalition structure $P=\left\{P_{1}, \ldots, P_{m}\right\}$ of $m$ coalitions several value functions have been proposed in the literature. The Aumann-Drèze value assigns to any player in a coalition $P_{k} \in P$ the Shapley value of the restriction

\footnotetext{
${ }^{1}$ For set-valued solutions in terms of share vectors we refer to van den Brink and van der Laan (1999b).
} 
of the game $(N, v)$ to coalition $P_{k}$, see Aumann and Drèze (1974). Under this value concept, the total payoff of the players within a coalition $P_{k}$ is equal to the worth of this coalition and therefore the total payoff is equal to $\sum_{k=1}^{m} v\left(P_{k}\right)$, which need not to be equal to $v(N)$. According to the Aumann-Drèze value, the payoff of a player $i$ in some coalition $P_{k}$ does not depend upon its contribution to any coalition containing players outside $P_{k}$. In fact, it is supposed that the grand coalition is not formed but that the players agree to disagree and are satisfied with cooperation within the coalitions $P_{k}, k=1, \ldots, m$. However, one can imagine many situations in which players form coalitions within the grand coalition. As already argued by Aumann and Drèze (1974), in many of such situations it is very reasonable that also the outside opportunities of the members of a coalition have to be taken into account. According to e.g. Hart and Kurz (1983) the existence of a coalition structure implies a two-level interaction between the players. Firstly, the worth of the grand coalition is distributed amongst the coalitions; secondly the payoff to each coalition is distributed amongst the players within this coalition. The outcome of such a two-level interaction is reflected by the so-called coalition structure value introduced by Owen (1977). The Owen coalition structure (CS-)value has the property that the total payoff of the players in a coalition $P_{k}$ is equal to the Shapley value of the coalition $P_{k}$, when this coalition is considered to be a player in the first level game between the coalitions. As a consequence we have that the Owen CS-value can be considered as a generalization of the Shapley value to games in coalition structure.

The Owen CS-share function is obtained by dividing the Owen CS-value by the worth $v(N)$ of the grand coalition (being equal to the sum of the Owen CS-values of all players). The Owen CS-share function alternatively can be defined using the following multiplication property. The Owen CS-share of player $i \in P_{k} \in P$ in the worth to be distributed is equal to the product of the Shapley share of coalition $P_{k}$ in the first level external game between the coalitions and the Shapley share of player $i$ in an appropriately defined second level internal game between the players in $P_{k}$. In the same way van der Laan and van den Brink (1999) define the Banzhaf CS-share function which assigns to player $i \in P_{k}$ the product of the Banzhaf share of coalition $P_{k}$ in the first level (external) game between coalitions and the Banzhaf share of player $i$ in an appropriately defined second level (internal) game.

In this paper we define a general class of CS-share functions using this multiplication property. All these CS-share functions satisfy the consistency property stating 
that the share of a coalition in the external game between a priori coalitions is equal to the sum of the shares of the players in this coalition in the original game in coalition structure. Moreover, we provide axiomatic characterizations of this class of CS-share functions using the multiplication and consistency properties. This class of CS-share functions generalizes the Owen CS-share function and the Banzhaf CS-share function. This Banzhaf CS-share function is not the same as the Banzhaf type CS-share function corresponding to the Banzhaf CS-value function introduced by Owen (1981) for games in coalition structure. In particular the share function obtained from Owen's Banzhaf CS-value function does neither satisfy the multiplication nor the consistency property.

The paper is organized as follows. In the next section we discuss some preliminaries on share functions and games in coalition structure. In Section 3 we introduce a class of share functions for games in coalition structure. In Section 4 we provide an axiomatic characterization of this class using the multiplication property. In Section 5 we characterize this class using consistency. Finally, we make some concluding remarks in Section 6.

\section{Preliminaries}

First, for player set $N$ and set $T \subset N$, the game $\left(N, u^{T}\right)$ denotes the unanimity game of coalition $T$, i.e. for $S \subset N$ we have that $u^{T}(S)=1$ if $T \subset S$ and $u^{T}(S)=0$ otherwise. Notice that $\left(N, u^{T}\right) \in \mathcal{G}$ for all $T \subset N$. For a pair of games $(N, v),(N, w) \in \mathcal{G}$ and real numbers $\alpha \geq 0$ and $\beta \geq 0$, the game $(N, z)$ with $z=\alpha v+\beta w$ is given by $z(E)=\alpha v(E)+\beta w(E)$ for all $E \subset N$. The set of monotone games is linear-closed, i.e. if $(N, v) \in \mathcal{G}$ and $(N, w) \in \mathcal{G}$, then also $(N, z) \in \mathcal{G}$ for all $\alpha, \beta \geq 0$. From Harsanyi $(1959,1963)$ we know that for any game $(N, v)$ it holds that

$$
v=\sum_{T \subset N} \Delta^{T}(v) u^{T}
$$

where the so-called Harsanyi-dividends $\Delta^{T}(v), T \subset N$, follow recursively from the systems of equations

$$
v(S)=\sum_{T \subset S} \Delta^{T}(v), \quad S \subset N
$$


It should be observed that a monotone game $(N, v) \in \mathcal{G}$ may have negative dividends, i.e. there may exist subsets $T$ with $\Delta^{T}(v)<0$. If so, for such a subset $T$ the game $\left(N, \Delta^{T}(v) u^{T}\right)$ is not in $\mathcal{G}$ and thus $v$ can not be written as a linear combination of monotone unanimity games. However, denoting

$$
v^{+}=\sum_{\left\{T \subset N \mid \Delta^{T}(v) \geq 0\right\}} \Delta^{T}(v) u^{T} \text { and } v^{-}=\sum_{\left\{T \subset N \mid \Delta^{T}(v)<0\right\}}-\Delta^{T}(v) u^{T}
$$

we have that $v+v^{-}=v^{+}$, showing that the monotone game $\left(N, v^{+}\right)$is equal to the sum of the two monotone games $(N, v)$ and $\left(N, v^{-}\right)$. This expression will be used in the proofs given in the Sections 4 and 5. Finally, for a game $(N, v)$ and a set $T \subset N$, the restricted game $\left(T, v_{T}\right)$ on player set $T$ is given by $v_{T}(E)=v(E)$ for all $E \subset T$.

\subsection{Share functions}

Let $m_{E}^{i}(N, v)=v(E)-v(E \backslash\{i\})$ be the marginal contribution of player $i \in N$ to coalition $E \subset N$ in game $(N, v) \in \mathcal{G}$. Then the well-known Shapley value $\varphi^{S}$ and Banzhaf value $\bar{\varphi}^{B}$ on the class $\mathcal{G}$ of monotone games are the functions defined by

$$
\varphi_{i}^{S}(N, v)=\sum_{\substack{E \subset N \\ E \ni i}} \widetilde{\omega}_{|E|}^{|N|} m_{E}^{i}(N, v), \text { and } \bar{\varphi}_{i}^{B}(N, v)=\sum_{\substack{E \subset N \\ E \ni i}} \widehat{\omega}_{|E|}^{|N|} m_{E}^{i}(N, v), \quad i \in N,
$$

where for $n \in \mathbb{N}$ and $k \in\{1, \ldots, n\}, \widetilde{\omega}_{k}^{n}=\frac{(k-1) !(n-k) !}{n !}$ and $\widehat{\omega}_{k}^{n}=\frac{1}{2^{n-1}}$. The Shapley value (Shapley (1953)) is characterized by the well-known axioms of efficiency, additivity, the null player property and symmetry. Axiomatizations of the Banzhaf value ${ }^{2}$ have been given by e.g. Lehrer (1988), Haller (1994), Nowak (1997) and Grabisch and Roubens (1999). Since the Banzhaf value is not efficient, this value is not adequate in allocating the worth $v(N)$ of the 'grand coalition'. To divide the worth $v(N)$ according to the Banzhaf value on the class of monotone non-null games van den Brink and van der Laan (1998) replace the Banzhaf value by the normalized Banzhaf value $\varphi^{B}$ which is the efficient value function that distributes the worth $v(N)$ proportional to the Banzhaf values of the players, i.e.

$$
\varphi_{i}^{B}(N, v)=\frac{\bar{\varphi}_{i}^{B}(N, v)}{\sum_{j \in N} \bar{\varphi}_{j}^{B}(N, v)} v(N), i \in N,(N, v) \in \mathcal{G}^{+},
$$

and $\varphi_{i}^{B}\left(N, v^{0}\right)=0$ for all $i \in N$.

\footnotetext{
${ }^{2}$ The Banzhaf value is introduced is as a power index for voting games by Banzhaf (1965), and is generalized to arbitrary games by, e.g. Owen (1975) and Dubey and Shapley (1979).
} 
An alternative approach to efficiently divide the worth of the grand coalition amongst its players is given by the concept of share function introduced by van der Laan and van den Brink (1998). A share function assigns to each player in a game its share in the worth to be distributed, i.e. a share function on a subclass $\mathcal{C} \subset \mathcal{G}$ of monotone games is a function $\rho$ on $\mathcal{C}$ satisfying $\sum_{i \in N} \rho_{i}(N, v)=1$ for all $(N, v) \in \mathcal{C}$. The Shapley share function $\rho^{S}$ on $\mathcal{G}^{+}$is given by

$$
\rho_{i}^{S}(N, v)=\frac{\varphi_{i}^{S}(N, v)}{v(N)} \text { for all } i \in N,(N, v) \in \mathcal{G}^{+} .
$$

The Banzhaf share function $\rho^{B}$ on $\mathcal{G}^{+}$is given by

$$
\rho_{i}^{B}(N, v)=\frac{\varphi_{i}^{B}(N, v)}{v(N)}=\frac{\bar{\varphi}_{i}^{B}(N, v)}{\sum_{j \in N} \bar{\varphi}_{j}^{B}(N, v)} \text { for all } i \in N,(N, v) \in \mathcal{G}^{+} .
$$

We now state the following properties for share functions. First, a share function $\rho$ satisfies the null player property on $\mathcal{C} \subset \mathcal{G}$ if for every $(N, v) \in \mathcal{C}$ and every null player ${ }^{3} i \in(N, v)$ it holds that $\rho_{i}(N, v)=0$. Second, $\rho$ satisfies symmetry on $\mathcal{C} \subset \mathcal{G}$ if for every $(N, v) \in \mathcal{C}$ and every pair $i, j$ of symmetric play$e r s^{4}$ in $(N, v)$ it holds that $\rho_{i}(N, v)=\rho_{j}(N, v)$. Finally, let be given some some real-valued function $\mu: \mathcal{G} \rightarrow \mathbf{R}$. Then $\rho$ satisfies $\mu$-additivity on $\mathcal{C} \subset \mathcal{G}$ if for every pair of games $(N, v),(N, w) \in \mathcal{C}$ satisfying $(N, v+w) \in \mathcal{C}$, it holds that $\mu(N, v+w) \rho(N, v+w)=\mu(N, v) \rho(N, v)+\mu(N, w) \rho(N, w)$. The last property is a generalization of the familiar additivity property which is obtained by taking $\mu(N, v)=1$ for all $(N, v) \in \mathcal{G}$.

Next we state some properties for functions $\mu: \mathcal{G} \rightarrow \mathbf{R}$. Let $\mathcal{C} \subset \mathcal{G}$. A function $\mu: \mathcal{C} \rightarrow \mathbf{R}$ is called positive on $\mathcal{C}$ if $\mu\left(N, v^{0}\right)=0$ and $\mu(N, v)>0$ for every $(N, v) \in$ $\mathcal{C} \cap \mathcal{G}^{+}$. We call $\mu$ additive on $\mathcal{C}$ if for every pair of games $(N, v),(N, w) \in \mathcal{C}$ satisfying $(N, v+w) \in \mathcal{C}$, it holds that $\mu(N, v+w)=\mu(N, v)+\mu(N, w)$. Finally, we call $\mu$ symmetric on $\mathcal{C}$ if for every $(N, v) \in \mathcal{C}$, every pair of symmetric players $i, j$ in $(N, v)$, and every $E \subset N$ such that $\{i, j\} \subset E$ and both restricted games $\left(E \backslash\{i\}, v_{E \backslash\{i\}}\right)$ and $\left(E \backslash\{j\}, v_{E \backslash\{j\}}\right)$ are in $\mathcal{C}$, it holds that $\mu\left(E \backslash\{i\}, v_{E \backslash\{i\}}\right)=\mu\left(E \backslash\{j\}, v_{E \backslash\{j\}}\right)$. The following two results follow from van der Laan and van den Brink (1998), see also van den Brink and van der Laan (1999a).

\footnotetext{
${ }^{3}$ Player $i \in N$ is a null player in $(N, v)$ if $v(E)=v(E \backslash\{i\})$ for all $E \subset N$.

${ }^{4}$ Players $i, j \in N$ are symmetric in $(N, v) \in \mathcal{G}$ if $v(E \cup\{i\})=v(E \cup\{j\})$ for all $E \subset N \backslash\{i, j\}$.
} 
Theorem 2.1 Let $\mu: \mathcal{G}^{+} \rightarrow \mathbf{R}$ be positive and symmetric on $\mathcal{G}^{+}$. Then there exists a unique share function $\rho^{\mu}$ on $\mathcal{G}^{+}$satisfying the null player property, symmetry and $\mu$-additivity on $\mathcal{G}^{+}$if and only if $\mu$ is additive on $\mathcal{G}^{+}$.

In case the $\mu$-function is a weighted sum of the marginal contributions of the players (with equal positive weights assigned to coalitions of equal size), then the corresponding share function is given in the next theorem.

Theorem 2.2 For given positive vectors $\omega^{n} \in \mathbf{R}_{+}^{n}, n \in \mathbb{N}$, let the function $\mu^{\omega}: \mathcal{G}^{+} \rightarrow$ $\mathbf{R}$ be defined by $\mu^{\omega}(N, v)=\sum_{i \in N} \sum_{E \ni i} \omega_{|E|}^{n} m_{E}^{i}(N, v)$. Then the unique share function satisfying the null player property, symmetry, and $\mu^{\omega}$-additivity on $\mathcal{G}^{+}$is given by

$$
\rho_{i}^{\mu^{\omega}}(N, v)=\frac{\sum_{\substack{E \subset N \\ E \ni i}} \omega_{|E|}^{n} m_{E}^{i}(N, v)}{\mu^{\omega}(N, v)} \text { for all } i \in N \text { and }(N, v) \in \mathcal{G}^{+} \text {. }
$$

Note that for any system of positive weights the corresponding function $\mu^{\omega}$ is positive, symmetric and additive on $\mathcal{G}^{+}$and thus satisfies the conditions of Theorem 2.1. In case the weight system $\omega^{n}$ is given by $\omega_{t}^{n}=\widetilde{\omega}_{t}^{n}=\frac{(n-t) !(t-1) !}{n !}, t=1, \ldots, n, n \in \mathbb{N}$, we denote $\mu^{S}=\mu^{\widetilde{\omega}}$ and we have that $\mu^{S}(N, v)=v(N)$. In case $\omega_{t}^{n}=\widehat{\omega}_{t}^{n}=2^{-(n-1)}, t=$ $1, \ldots, n, n \in \mathbb{N}$, we denote $\mu^{B}=\mu^{\widehat{\omega}}$ and it follows that $\mu^{B}(N, v)=\frac{1}{2^{n-1}} \sum_{E \subset N}(2|E|-$ $n) v(E)$. In van der Laan and van den Brink (1998) it is shown that the unique share function satisfying the properties stated in Theorem 2.2 with $\mu^{\omega}=\mu^{S}$ is the Shapley share function $\rho^{S}$, and the unique share function satisfying these properties with $\mu^{\omega}=\mu^{B}$ is the Banzhaf share function $\rho^{B}$.

\subsection{Games in coalition structure}

The share functions defined in the previous section yield a distribution of the worth of the grand coalition reflecting the individual bargaining position of the players. In many situations however, it is reasonable to suppose that players form coalitions which decide to act together against the other players in bargaining over $v(N)$. In this section we consider situations in which the players are organized in a priori given coalition structure.

A coalition structure on a player set $N$ is a finite partition $P=\left\{P_{1}, \ldots, P_{m}\right\}$ of $m$ non-empty, disjoint subsets of $N$, i.e. $\cup_{k=1}^{m} P_{k}=N$ and $P_{k} \cap P_{\ell}=\emptyset$ for all $k, \ell \in\{1, \ldots, m\}, k \neq \ell$. In the following the set of coalitions in the coalition 
structure $P=\left\{P_{1}, \ldots, P_{m}\right\}$ is denoted by $M=\{1, \ldots, m\}$ with $k \in M$ representing coalition $P_{k} \in P$. Furthermore, a monotone game $(N, v) \in \mathcal{G}$ in coalition structure $P$ is denoted by $(N, v, P)$. The collection of all coalition structures on $N$ is denoted by $\mathcal{P}^{N}$. The collection of all monotone games in coalition structure is denoted by $\mathcal{G P}$. A coalition structure (CS-)value function $\theta$ on the set $\mathcal{G P}$ assigns a payoff to any player in every monotone game in coalition structure $(N, v, P)$. Hart and Kurz (1983) argue that the existence of a coalition structure implies a two-level interaction between the players. The outcome of such a two-level interaction is reflected by the Owen Coalition Structure value (Owen CS-value) $\theta^{S}$ introduced by Owen (1977), which is defined by

$$
\begin{aligned}
\theta_{i}^{S}(N, v, P)= & \sum_{\substack{L \subset M \\
L \not \supset k}} \sum_{\substack{E \subset P_{k} \\
E \ni i}} \frac{|L| !(m-|L|-1) !}{m !} \cdot \frac{(|E|-1) !\left(\left|P_{k}\right|-|E|\right) !}{\left|P_{k}\right| !} \times \\
& (v(E \cup P(L))-v((E \backslash\{i\}) \cup P(L))), i \in P_{k} \in P, k \in M,
\end{aligned}
$$

where $P(L)=\cup_{j \in L} P_{j}$. We remark that the Owen CS-value reduces to the Shapley value when $P=\{N\}$ or when $P=\left\{\{i\}_{i \in N}\right\}$. The weights of the marginal values are a product of two 'Shapley weights', reflecting the fact that first coalitions enter subsequently in a random order and that within each coalition the players enter subsequently in a random order.

For given $(N, v, P) \in \mathcal{G P}$, with $P=\left\{P_{1}, \ldots, P_{m}\right\}$, the $m$-player first-level or external game between coalitions is the game $\left(M, v^{P}\right) \in \mathcal{G}$ defined by $M=\{1, \ldots, m\}$ as the set of players and characteristic function $v^{P}$ given by

$$
v^{P}(L)=v(P(L)), \quad L \subset M
$$

This game is already introduced by Owen (1977) who calles it the quotient game. For all $k \in M$, it follows from equation (1) by summing up over all $i \in P_{k}$ that

$$
\sum_{i \in P_{k}} \theta_{i}^{S}(N, v, P)=\theta_{k}^{S}\left(M, v^{P},\{M\}\right)=\varphi_{k}^{S}\left(M, v^{P}\right),
$$

showing that the sum of the payoffs to the players in $P_{k}$ is equal to the Shapley value of player $k$ (representing coalition $P_{k}$ ) in the external game between the coalitions. Since this property generalizes the fact that the Owen CS-value reduces to the Shapley value when $P=\{N\}$ or when $P=\left\{\{i\}_{i \in N}\right\}$, as mentioned already above, we say that the Owen CS-value is consistent. Since by efficiency of the Shapley value 
$\sum_{k \in M} \varphi_{k}^{S}\left(M, v^{P}\right)=v^{P}(M)=v(N)$, it follows from equation (2) that also the Owen CS-value is efficient. For axiomatizations of the Owen CS-value we refer to Owen (1977), Hart and Kurz (1983) and Winter (1989).

A $C S$-share function is a function $\psi$ on $\mathcal{G P}$ satisfying $\sum_{i \in N} \psi_{i}(N, v, P)=1$ for all $(N, v, P) \in \mathcal{G} \mathcal{P}$ and assigns to every player in a monotone game in coalition structure a share in the worth $v(N)$ to be distributed. The Owen $C S$-share function assigns to every player in a monotone game in coalition structure its share according to the Owen CS-value, i.e. it is the function $\psi^{S}$ given by

$$
\psi_{i}^{S}(N, v, P)=\frac{\theta_{i}^{S}(N, v, P)}{\sum_{j \in N} \theta_{j}^{S}(N, v, P)}=\frac{\theta_{i}^{S}(N, v, P)}{v(N)}, \quad i \in N .
$$

The Owen CS-share of player $i \in P_{k} \in P$ can be reformulated as the product of two Shapley shares. The first share is the share $\rho_{k}^{S}\left(M, v^{P}\right)$ of coalition $k$ in the $m$-player first-level external game $\left(M, v^{P}\right)$ between coalitions that is discussed before. The second share is the share $\rho_{i}^{S}\left(P_{k}, v^{P_{k}}\right)$ of player $i$ in the $\left|P_{k}\right|$-player second-level or internal game $\left(P_{k}, v^{P_{k}}\right)$ given by

$$
v^{P_{k}}(E)=\sum_{\substack{L \subset M \\ L \not \nexists k}} \frac{|L| !(m-|L|-1) !}{m !} v^{P_{k}, L}(E), \quad E \subset P_{k},
$$

where, for $L \subset M$ and $k \notin L$,

$$
v^{P_{k}, L}(E)=v(E \cup P(L))-v(P(L))
$$

is the marginal contribution of $E \subset P_{k}$ to the union $P(L)$ of the coalitions $P_{j}, j \in L$. So, the internal game $\left(P_{k}, v^{P_{k}}\right)$ is a weighted sum of the marginal contributions $v^{P_{k}, L}$, $L \subset M$, where the weight of the marginal contribution $v^{P_{k}, L}$ is equal to the Shapley weight $\widetilde{\omega}_{|L|+1}^{m}=\frac{|L| !(m-|L|-1) !}{m !}$ assigned to coalition $k \in M$ if this coalition joins the collection $L \subset M$ of coalitions. For given $(N, v, P) \in \mathcal{G P}$ with $P=\left\{P_{1}, \ldots, P_{m}\right\}$ and $M=\{1, \ldots, m\}$ van der Laan and van den Brink (1999) show that ${ }^{5}$

$$
\psi_{i}^{S}(N, v, P)=\rho_{i}^{S}\left(P_{k}, v^{P_{k}}\right) \cdot \rho_{k}^{S}\left(M, v^{P}\right), i \in P_{k}, k \in M
$$

Van der Laan and van den Brink (1999) use this multiplication property to define a Banzhaf-type coalition structure share function. To do so they replace in equation (3)

\footnotetext{
${ }^{5}$ Owen (1977) shows a similar property without using the concept of share function.
} 
the second-level Shapley weights by the corresponding second-level Banzhaf weights $\widehat{\omega}_{|L|+1}^{m}=2^{-(m-1)}$ to obtain the $\left|P_{k}\right|$-player internal game $\left(P_{k}, \widehat{v}^{P_{k}}\right), k \in M$, defined by

$$
\widehat{v}^{P_{k}}(E)=\sum_{\substack{L \subset M \\ L \not \supset k}} 2^{-(m-1)} v^{P_{k}, L}(E), \quad E \subset P_{k} .
$$

Applying a similar multiplication property as (5) they define the Banzhaf Coalition Structure (CS-)share function as the function $\psi^{B}$ on $\mathcal{G P}$ given by

$$
\psi_{i}^{B}(N, v, P)=\rho_{i}^{B}\left(P_{k}, \widehat{v}^{P_{k}}\right) \cdot \rho_{k}^{B}\left(M, v^{P}\right), i \in P_{k}, k \in M
$$

This Banzhaf CS-share function is different from the CS-share function that is obtained by normalizing the Banzhaf-type CS-value function that is introduced in Owen (1981) (see also Section 6).

\section{A class of CS-share functions for games in coali- tion structure}

In this section we generalize the share functions $\rho^{\mu}$ discussed in Section 2.1 to games in coalition structure. The share functions $\rho^{\mu}$ are defined on the class $\mathcal{G}^{+}$of monotone non-null games. For null games the concept of share function is in itself not very interesting, because in such a game it seems reasonable that any player gets a payoff of zero irrespective of the shares. Moreover, in many applications we may restrict ourselves to the class $\mathcal{G}^{+}$of monotone non-null games. However, in this paper we apply the concept of share functions to games in coalition structure. As argued before, for such games the payoff of a player can be seen as the result of a first-level external game between coalitions and a second-level internal game between the players within a coalition. In such a set-up we have to deal with null games, which may appear on the second (internal) level, even when the game itself is a non-null game. Therefore we extend the concept of share functions to null games by giving all players an equal share, i.e.

$$
\rho^{\mu}\left(N, v^{0}\right)=\frac{1}{|N|} \text { for all } i \in N .
$$

We can generalize Theorem 2.1 to the class $\mathcal{G}$ of all monotone games in a straightforward way by requiring symmetry and $\mu$-additivity to hold for all games in $\mathcal{G}$, and 
requiring the null player property only for the non-null games ${ }^{6}$ in $\mathcal{G}^{+}$.

We generalize the share functions $\rho^{\mu}$ to games in coalition structure using the multiplication property that defines the share for player $i \in P_{k}$ in game $(N, v)$ in coalition structure $P=\left\{P_{1}, \ldots, P_{m}\right\}$, as the product of the share of coalition $k$ in the external game $\left(M, v^{P}\right)$ in which the coalitions in $P$ act as individual players, and the share of player $i$ in some internal game among the players in $P_{k}$. To do so, for given positive, additive and symmetric function $\mu: \mathcal{G} \rightarrow \mathbf{R}$, game $(N, v) \in \mathcal{G}$ and coalition structure $P \in \mathcal{P}^{N}$, we define for $P_{k} \in P$ the $\mu$-internal game $\left(P_{k}, v_{\mu}^{P_{k}}\right)$ by

$$
v_{\mu}^{P_{k}}(E)=\rho_{k}^{\mu}\left(M, v_{P_{k} \mid E}^{P}\right) \cdot v(P(M \backslash\{k\}) \cup E), \quad E \subset P_{k},
$$

where $M=\{1, \ldots, m\}$ and the game $\left(M, v_{P_{k} \mid E}^{P}\right)$ on the player set $M$ of coalitions is given by the characteristic function $v_{P_{k} \mid E}^{P}, E \subset P_{k} \in P$, defined by

$$
v_{P_{k} \mid E}^{P}(L)=\left\{\begin{array}{cl}
v(P(L \backslash\{k\}) \cup E) & \text { if } k \in L \subset M, \\
v(P(L)) & \text { if } k \notin L \subset M .
\end{array}\right.
$$

So, the characteristic function $v_{P_{k} \mid E}^{P}$ assigns to every coalition $L$ of coalitions in the coalition structure the worth of the union of these coalitions where coalition $P_{k}$ is replaced by $E \subset P_{k}$, i.e. player $l \in M \backslash\{k\}$ represents a priori coalition $P_{l} \neq P_{k}$ in the coalition structure $P$, whereas player $k$ represents the subcoalition $E$ of $P_{k}$ instead of $P_{k}$ as a whole. Notice that this game also appears in Owen (1977), reflecting in some sense coalition's $E \subset P_{k}$ possibilities if it defects from $P_{k}$.

Given a positive, additive and symmetric function $\mu: \mathcal{G} \rightarrow \mathbf{R}$, in the next theorem we introduce a function $\psi^{\mu}$ on $\mathcal{G}$ using the multiplication property with respect to the external game $\left(M, v^{P}\right)$ and the internal game $\left(P_{k}, v_{\mu}^{P_{k}}\right)$ and show that this function is a share function satisfying a set of consistency properties.

Theorem 3.1 Let $\mu: \mathcal{G} \rightarrow \mathbf{R}$ be positive, additive and symmetric on $\mathcal{G}$ and let $\rho^{\mu}$ be the share function as defined in Theorem 2.1 extended by (7). Then the function $\psi^{\mu}$ on $\mathcal{G P}$ defined by

$$
\psi_{i}^{\mu}(N, v, P)=\rho_{i}^{\mu}\left(P_{k}, v_{\mu}^{P_{k}}\right) \cdot \rho_{k}^{\mu}\left(M, v^{P}\right),
$$

for $i \in P_{k} \in P=\left\{P_{1}, \ldots, P_{m}\right\} \in \mathcal{P}^{N}, k \in M=\{1, \ldots, m\}$, is a CS-share function and satisfies for every $(N, v, P) \in \mathcal{G P}$ the following consistency properties:

\footnotetext{
${ }^{6}$ Clearly, the null player property is not satisfied by any share function on a class of games that contains null games. Note that symmetry and the fact that CS-shares by definition add up to one, imply the equal share distribution for null games.
} 
1. $\sum_{i \in P_{k}} \psi_{i}^{\mu}(N, v, P)=\rho_{k}^{\mu}\left(M, v^{P}\right)$;

2. $\psi^{\mu}(N, v, P)=\rho^{\mu}(N, v)$ when $P=\{N\}$;

3. $\psi^{\mu}(N, v, P)=\rho^{\mu}(N, v)$ when $P=\left\{\{i\}_{i \in N}\right\}$.

\section{PROOF}

Since $\rho^{\mu}$ satisfies $\sum_{i \in N} \rho_{i}^{\mu}(N, v)=1$, it follows that

$$
\begin{aligned}
\sum_{i \in N} \psi_{i}^{\mu}(N, v, P) & =\sum_{k \in M} \sum_{i \in P_{k}} \psi_{i}^{\mu}(N, v, P) \\
& =\sum_{k \in M} \sum_{i \in P_{k}} \rho_{i}^{\mu}\left(P_{k}, v_{\mu}^{P_{k}}\right) \cdot \rho_{k}^{\mu}\left(M, v^{P}\right)=\sum_{k \in M} \rho_{k}^{\mu}\left(M, v^{P}\right)=1
\end{aligned}
$$

and thus $\psi^{\mu}$ is indeed a CS-share function. It further follows that

1. $\sum_{i \in P_{k}} \psi_{i}^{\mu}(N, v, P)=\sum_{i \in P_{k}} \rho_{i}^{\mu}\left(P_{k}, v_{\mu}^{P_{k}}\right) \cdot \rho_{k}^{\mu}\left(M, v^{P}\right)=\rho_{k}^{\mu}\left(M, v^{P}\right)$.

2. For $P=\left\{P_{1}\right\}$ with $P_{1}=N$, and $M=\{1\}$ it follows with equation (8) that $v_{\mu}^{N}(E)=\rho_{1}^{\mu}\left(\{1\}, v_{P_{1} \mid E}^{P}\right) \cdot v(E)=v(E)$ for $E \subset P_{1}=N$. Since $v_{\mu}^{N}=v$ and $\rho_{1}^{\mu}\left(\{1\}, v^{P}\right)=1$, it follows that $\psi_{i}^{\mu}(N, v,\{N\})=\rho_{i}^{\mu}\left(N, v_{\mu}^{N}\right) \cdot \rho_{1}^{\mu}\left(\{1\}, v^{P}\right)=$ $\rho_{i}^{\mu}(N, v)$ for every $i \in N$.

3. If $P=\left\{\{i\}_{i \in N}\right\}$ then $M=N$ and $v^{P}(L)=v(P(L))=v(L)$ for all $L \subset M=N$. Since $\rho_{i}^{\mu}\left(\{i\}, v_{\mu}^{\{i\}}\right)=1$ and $\left(M, v^{P}\right)=(N, v)$, it follows that $\psi_{i}^{\mu}\left(N, v,\left\{\{i\}_{i \in N}\right\}\right)=$ $\rho_{i}^{\mu}\left(\{i\}, v_{\mu}^{\{i\}}\right) \cdot \rho_{i}^{\mu}\left(M, v^{P}\right)=\rho_{i}^{\mu}(N, v)$ for every $i \in N$.

Q.E.D.

The consistency properties 2 and 3 in this theorem show that $\psi^{\mu}$ generalizes the share functions $\rho^{\mu}$ of Theorem 2.1 extended by (7), i.e. for any game $(N, v) \in \mathcal{G}$ we have that $\rho^{\mu}(N, v)=\psi^{\mu}(N, v,\{N\})=\psi^{\mu}\left(N, v,\left\{\{i\}_{i \in N}\right\}\right)$. These two consistency properties also imply that property 1 can be written as $\sum_{i \in P_{k}} \psi_{i}^{\mu}(N, v, P)=$ $\psi_{k}^{\mu}\left(M, v^{\{M\}},\{M\}\right)=\psi_{k}^{\mu}\left(M, v^{\left\{\{k\}_{k \in M}\right\}},\left\{\{k\}_{k \in M}\right\}\right)$.

The next proposition shows that the function $\psi^{\mu}$ is the Owen CS-share function $\psi^{S}$, when taking the Shapley $\mu$-function $\mu^{S}$, while $\psi^{\mu}$ is the Banzhaf CS-share function $\psi^{B}$, when taking the Banzhaf $\mu$-function $\mu^{B}$.

Proposition 3.2 The CS-share function $\psi^{\mu}$ is the Owen $C S$-share function $\psi^{S}$ when $\mu=\mu^{S}=v(N)$ and it is the Banzhaf CS-share function $\psi^{B}$ when $\mu=\mu^{B}(N, v)=$ $\frac{1}{2^{n-1}} \sum_{E \subset N}(2|E|-n) v(E)$. 


\section{PROOF}

According to equation (5) we have that $\psi_{i}^{S}(N, v, P)=\rho_{i}^{S}\left(P_{k}, v^{P_{k}}\right) \cdot \rho_{k}^{S}\left(M, v^{P}\right)$, while according to Theorem 3.1 the function $\psi^{\mu^{S}}$ is given by $\psi_{i}^{\mu^{S}}(N, v, P)=\rho_{i}^{\mu^{S}}\left(P_{k}, v_{\mu^{S}}^{P_{k}}\right)$. $\rho_{k}^{\mu^{S}}\left(M, v^{P}\right)$, for $i \in P_{k} \in P$. Since $\rho^{S}=\rho^{\mu^{S}}$ (see van der Laan and van den Brink (1998)), it is sufficient to show that the internal game $\left(P_{k}, v^{P_{k}}\right)$ as given in equation (3) is equal to the internal game $\left(P_{k}, v_{\mu^{S}}^{P_{k}}\right)$ as given in equation (8). Since $v_{P_{k} \mid E}^{P}(M)=v(P(M \backslash\{k\}) \cup E)$, it follows that $v_{\mu^{S}}^{P_{k}}(E)=\rho_{k}^{S}\left(M, v_{P_{k} \mid E}^{P}\right)$. $v(P(M \backslash\{k\}) \cup E)=\varphi_{k}^{S}\left(M, v_{P_{k} \mid E}^{P}\right)=\sum_{L \not \subset k} \frac{|L| !(m-|L|-1) !}{m !}\left(v_{P_{k} \mid E}^{P}(L \cup\{k\})-v_{P_{k} \mid E}^{P}(L)\right)=$ $\sum_{\substack{L \subset M \\ L \not k}} \frac{|L| !(m-|L|-1) !}{m !} v(P(L) \cup E)-v(P(L))=\sum_{\substack{L \subset M \\ L \not k}} \frac{|L| !(m-|L|-1) !}{m !} v^{P_{k}, L}(E)=v^{P_{k}}(E)$ for all $E \subset P_{k}$.

Analogously, for the Banzhaf CS-share function we can show that the internal game $\left(P_{k}, \widehat{v}^{P_{k}}\right)$ as given in equation (6) is equal to the internal game $\left(P_{k}, v_{\mu^{B}}^{P_{k}}\right)$. Q.E.D.

Other CS-share functions are obtained by taking other $\mu$-functions. For instance, consider the non-efficient Deegan-Packel value given by $\varphi_{i}^{D P}(N, v)=\sum_{\substack{E \subset N \\ E \ni i}} \frac{v(E)}{|E|}$ for all $i \in$ $N$, see Deegan and Packel (1979), and let the corresponding share function $\rho^{D P}$ be given by $\rho_{i}^{D P}(N, v)=\frac{\varphi_{i}^{D P}(N, v)}{\sum_{j \in N} \varphi_{j}^{D P}(N, v)}$ for all $i \in N$. This share function satisfies the axioms of symmetry and $\mu^{D P}$-additivity with $\mu^{D P}(N, v)=\sum_{E \subset N} v(E)$, but does not satisfy the null player property and thus does not belong to the class of share functions given in Theorem 2.1. However, since $\mu^{D P}$ is positive, additive and symmetric on $\mathcal{G}$, according to Theorem 2.1 there exists a unique share function on $\mathcal{G}^{+}$satisfying symmetry, $\mu^{D P}$-additivity and the null player property. Van der Laan and van den Brink (1998) show that this is the share function $\rho^{\mu^{D P}}$ that is obtained as in Theorem 2.2 by taking positive weight vectors $\bar{\omega}^{n} \in \mathbf{R}_{+}^{n}, n \in \mathbb{N}$, recursively given by $\bar{\omega}_{n}^{n}=\frac{1}{n}$ and $\bar{\omega}_{t}^{n}=\frac{1+(n-t) \bar{\omega}_{t+1}^{n}}{t}, t=n-1, \ldots, 1$. Applying this solution to games in coalition structure as defined in Theorem 3.1 gives the CS-share function $\psi^{\mu^{D P}}$ given by

$$
\psi_{i}^{\mu^{D P}}(N, v, P)=\rho_{i}^{\mu^{D P}}\left(P_{k}, v_{\mu^{D P}}^{P_{k}}\right) \cdot \rho_{k}^{\mu^{D P}}\left(M, v^{P}\right) .
$$

In the next example we evaluate the Owen CS-share function, the Banzhaf CS-share function and this $\mu^{D P}$ CS-share function for a four player game with a three coalition structure.

Example 3.3 Consider the game $(N, v)$ on $N=\{1,2,3,4\}$ given by $v(S)=1$ if $1 \in$ $S$ and $|S| \geq 2, v(S)=0$ otherwise. The Shapley, Banzhaf and $\mu^{D P}$-shares of the players in this game are given by $\rho^{S}(N, v)=\left(\frac{3}{4}, \frac{1}{12}, \frac{1}{12}, \frac{1}{12}\right)^{\top}, \rho^{B}(N, v)=\left(\frac{7}{10}, \frac{1}{10}, \frac{1}{10}, \frac{1}{10}\right)^{\top}$ 


\begin{tabular}{|c||c|c|c|c|}
\hline$E \subset P_{1}$ & $v^{P_{1}, \emptyset}(E)$ & $v^{P_{1},\{2\}}(E)$ & $v^{P_{1},\{3\}}(E)$ & $v^{P_{1},\{2,3\}}(E)$ \\
\hline$\emptyset$ & 0 & 0 & 0 & 0 \\
$\{1\}$ & 0 & 1 & 1 & 1 \\
$\{2\}$ & 0 & 0 & 0 & 0 \\
$\{1,2\}$ & 1 & 1 & 1 & 1 \\
\hline
\end{tabular}

Table 1: Marginal contributions $v^{P_{1}, L}(E), L \subset\{2,3\} \subset M, E \subset P_{1}=\{1,2\}$

\begin{tabular}{|c||c|c|c|c|}
\hline & $E=\emptyset$ & $E=\{1\}$ & $E=\{2\}$ & $E=\{1,2\}$ \\
\hline$\mu=\mu^{S}$ & 0 & $\frac{2}{3}$ & 0 & 1 \\
$\mu=\mu^{B}$ & 0 & $\frac{3}{4}$ & 0 & 1 \\
$\mu=\mu^{D P}$ & 0 & $\frac{5}{3}$ & 0 & 4 \\
\hline
\end{tabular}

Table 2: Characteristic functions of the internal games $v_{\mu}^{P_{1}}$

and $\rho^{\mu^{D P}}(N, v)=\left(\frac{51}{84}, \frac{11}{84}, \frac{11}{84}, \frac{11}{84}\right)^{\top}$. They are obtained from applying Theorem 2.2 with weight vector $\widetilde{\omega}^{4}=\left(\frac{1}{4}, \frac{1}{12}, \frac{1}{12}, \frac{1}{4}\right)^{\top}$ for $\rho^{S}$, $\widehat{\omega}^{4}=\left(\frac{1}{8}, \frac{1}{8}, \frac{1}{8}, \frac{1}{8}\right)^{\top}$ for $\rho^{B}$, respectively $\bar{\omega}^{4}=\left(\frac{45}{12}, \frac{11}{12}, \frac{5}{12}, \frac{1}{4}\right)^{\top}$ for $\rho^{\mu^{D P}}$.

For coalition structure $P=\left\{P_{1}, P_{2}, P_{3}\right\}$ with $P_{1}=\{1,2\}, P_{2}=\{3\}$ and $P_{3}=\{4\}$, the external game $\left(M, v^{P}\right)$ with $M=\{1,2,3\}$ is given by $v^{P}(S)=1$ if $1 \in S$ and $v^{P}(S)=0$ otherwise. Then $\rho^{S}\left(M, v^{P}\right)=\rho^{B}\left(M, v^{P}\right)=\rho^{\mu^{D P}}\left(M, v^{P}\right)=(1,0,0)^{\top}$. Since the Shapley-, Banzhaf- and $\mu^{D P}$-shares of the coalitions $\{3\}$ and $\{4\}$ in the external game are zero, it follows that the Shapley-, Banzhaf-, and $\mu^{D P}$-CS-shares of players 3 and 4 are equal to 0 . So, we are left to determine the CS-shares for players 1 and 2. Using for $L \subset\{2,3\} \subset M$ the marginal contributions $v^{P_{1}, L}(E), E \subset P_{1}$, (see equation (4)) as given in Table 1 , and the weight vectors $\widetilde{\omega}^{3}=\left(\frac{1}{3}, \frac{1}{6}, \frac{1}{3}\right)^{\top}, \widehat{\omega}^{3}=$ $\left(\frac{1}{4}, \frac{1}{4}, \frac{1}{4}\right)^{\top}$ and $\bar{\omega}^{3}=\left(\frac{7}{3}, \frac{2}{3}, \frac{1}{3}\right)^{\top}$, this gives the internal games $v_{\mu}^{P_{1}}$ given in Table 2.

Finally, with $\widetilde{\omega}^{2}=\widehat{\omega}^{2}=\left(\frac{1}{2}, \frac{1}{2}\right)^{\top}$ and $\widetilde{\omega}^{2}=\left(\frac{3}{4}, \frac{1}{2}\right)^{\top}$ we obtain $\rho^{S}\left(P_{1}, v_{\mu^{S}}^{P_{1}}\right)=\left(\frac{5}{6}, \frac{1}{6}\right)^{\top}$, $\rho^{B}\left(P_{1}, v_{\mu^{B}}^{P_{1}}\right)=\left(\frac{7}{8}, \frac{1}{8}\right)^{\top}$ and $\rho^{\mu^{D P}}\left(P_{1}, v_{\mu^{D P}}^{P_{1}}\right)=\left(\frac{27}{34}, \frac{7}{34}\right)^{\top}$. So, the Shapley-, Banzhaf- and $\mu^{D P}$-CS-shares are given by $\psi^{S}(N, v, P)=\left(\frac{5}{6}, \frac{1}{6}, 0,0\right)^{\top}, \psi^{B}(N, v, P)=\left(\frac{7}{8}, \frac{1}{8}, 0,0\right)^{\top}$, respectively, $\psi^{\mu^{D P}}(N, v, P)=\left(\frac{27}{34}, \frac{7}{34}, 0,0\right)^{\top}$. $\quad$ Q.E.D. 


\section{An axiomatization of CS-share functions using the multiplication property}

In this section we provide an axiomatization of the CS-share functions defined in Theorem 3.1 using the multiplication property. So, for $\mu$ a symmetric, additive and positive function on $\mathcal{G}$, we state several axioms for a share function $\psi$ on $\mathcal{G P}$. Since we want to axiomatically characterize CS-share functions for games in coalition structure we state all axioms in terms of CS-shares functions.

In the next axiom it is required that a share function satisfies the multiplication property. Since in this property the internal game $v_{\mu}^{P_{k}}$ appears and this game depends on the chosen $\mu$-function, the multiplication property also depends on this $\mu$-function.

Axiom 4.1 ( $\mu$-Multiplication) For $\mu: \mathcal{G} \rightarrow \mathbf{R},(N, v, P) \in \mathcal{G P}$ and $i \in P_{k} \in P=$ $\left\{P_{1}, \ldots, P_{m}\right\}$ it holds that

$$
\psi_{i}(N, v, P)=\psi_{i}\left(P_{k}, v_{\mu}^{P_{k}},\left\{P_{k}\right\}\right) \cdot \psi_{k}\left(M, v^{P},\{M\}\right) .
$$

The null player property is a straightforward generalization of the null player property for share functions.

Axiom 4.2 (Null player property) If $i \in N$ is a null player in $(N, v) \in \mathcal{G}^{+}$then $\psi_{i}(N, v, P)=0$ for every $P \in \mathcal{P}^{N}$.

Note that the null player property only requires that null players in monotone nonnull games earn a zero payoff.

As done in Winter $(1989,1992)$ for CS-value functions, we distinguish two symmetry axioms for CS-share functions: an individual and a coalitional symmetry axiom. The individual symmetry axiom states that players who belong to the same a priori coalition and are symmetric in the game earn the same share in the payoff. (Coalitional symmetry is introduced after the next theorem.)

Axiom 4.3 (Individual symmetry) If $i, j \in P_{k} \in P \in \mathcal{P}^{N}$ are symmetric in $(N, v) \in \mathcal{G}$ then $\psi_{i}(N, v, P)=\psi_{j}(N, v, P)$.

In property 2 of Theorem 3.1 we saw that the CS-share function $\psi^{\mu}$ generalizes the share functions $\rho^{\mu}$ by taking coalition structure $P=\{N\}$. In Section 2 we already mentioned that the share functions $\rho^{\mu}$ satisfy $\mu$-additivity. Next we restate $\mu$-additivity for games in coalition structure in terms of CS-share functions. 
Axiom 4.4 ( $\mu$-additivity for one-coalition structures) Let $\mu: \mathcal{G} \rightarrow \mathbf{R}$ be given. For every $(N, v),(N, w) \in \mathcal{G}$ it holds that

$$
\mu(N, v+w) \psi(N, v+w,\{N\})=\mu(N, v) \psi(N, v,\{N\})+\mu(N, w) \psi(N, w,\{N\}) .
$$

The next theorem proves that the CS-share function $\psi^{\mu}$ given in Theorem 3.1 is the unique CS-share functions satisfying the four axioms stated above.

Theorem 4.5 Let $\mu: \mathcal{G} \rightarrow \mathbf{R}$ be positive, additive and symmetric on $\mathcal{G}$. Then $\psi^{\mu}$ is the unique $C S$-share function on $\mathcal{G}$ that satisfies $\mu$-multiplication, the null player property, individual symmetry and $\mu$-additivity for one-coalition structures.

\section{ProOF}

The property of $\mu$-multiplication of $\psi^{\mu}$ follows directly from the definition of $\psi^{\mu}$ and consistency property 2 in Theorem 3.1. Next, let $i \in P_{k} \in P \in \mathcal{P}^{N}$ be a null player in $(N, v) \in \mathcal{G}^{+}$. Then $v(P(M \backslash\{k\}) \cup E)=v(P(M \backslash\{k\}) \cup(E \backslash\{i\}))$ for all $E \subset P_{k}, E \ni i$, and $v_{P_{k} \mid E}^{P}(L)=v_{P_{k} \mid E \backslash\{i\}}^{P}(L)$ for all $L \subset M$. This implies that $v_{\mu}^{P_{k}}(E)-v_{\mu}^{P_{k}}(E \backslash\{i\})=\rho_{k}^{\mu}\left(M, v_{P_{k} \mid E}^{P}\right) \cdot v(P(M \backslash\{k\}) \cup E)-\rho_{k}^{\mu}\left(M, v_{P_{k} \mid E \backslash\{i\}}^{P}\right) \cdot v(P(M \backslash$ $\{k\}) \cup(E \backslash\{i\}))=0$ for all $E \subset P_{k}, E \ni i$. Thus, $i$ is a null player in $\left(P_{k}, v_{\mu}^{P_{k}}\right)$. Since $\rho^{\mu}$ satisfies the null player property we have that $\rho_{i}^{\mu}\left(P_{k}, v_{\mu}^{P_{k}}\right)=0$, and thus $\psi_{i}^{\mu}(N, v, P)=\rho_{i}^{\mu}\left(P_{k}, v_{\mu}^{P_{k}}\right) \cdot \rho_{k}^{\mu}\left(M, v^{P}\right)=0$, if $\left(P_{k}, v_{\mu}^{P_{k}}\right) \in \mathcal{G}^{+}$. If $\left(P_{k}, v_{\mu}^{P_{k}}\right)$ is a null game then $v_{\mu}^{P_{k}}\left(P_{k}\right)=\rho_{k}^{\mu}\left(M, v_{P_{k} \mid P_{k}}^{P}\right) \cdot v(N)=0$. Since $(N, v) \in \mathcal{G}^{+}$we have that $v(N)>0$, and thus $\rho_{k}^{\mu}\left(M, v_{P_{k} \mid P_{k}}^{P}\right)$ must be equal to 0 . Since $v_{P_{k} \mid P_{k}}^{P}=v^{P}$ it then holds that $\rho_{k}^{\mu}\left(M, v^{P}\right)=0$. Again $\psi_{i}^{\mu}(N, v, P)=\rho_{i}^{\mu}\left(P_{k}, v_{\mu}^{P_{k}}\right) \cdot \rho_{k}^{\mu}\left(M, v^{P}\right)=0$. Hence, $\psi^{\mu}$ satisfies the null player property.

If $i, j \in P_{k}$ are symmetric in $(N, v)$ then $i, j \in P_{k}$ are symmetric in $\left(P_{k}, v_{\mu}^{P_{k}}\right)$. Symmetry of $\rho^{\mu}$ then implies that $\psi_{i}^{\mu}(N, v, P)=\rho_{i}^{\mu}\left(P_{k}, v_{\mu}^{P_{k}}\right) \cdot \rho_{k}^{\mu}\left(M, v^{P}\right)=\rho_{j}^{\mu}\left(P_{k}, v_{\mu}^{P_{k}}\right)$. $\rho_{k}^{\mu}\left(M, v^{P}\right)=\psi_{j}^{\mu}(N, v, P)$.

Finally, the consistency property 2 of Theorem 3.1 and $\mu$-additivity of $\rho^{\mu}$ imply that $\mu(N, v+w) \psi^{\mu}(N, v+w,\{N\})=\mu(N, v+w) \rho^{\mu}(N, v+w)=\mu(N, v) \rho^{\mu}(N, v)+$ $\mu(N, w) \rho^{\mu}(N, w)=\mu(N, v) \psi^{\mu}(N, v,\{N\})+\mu(N, w) \psi^{\mu}(N, w,\{N\})$, which shows that $\psi^{\mu}$ satisfies $\mu$-additivity for one-coalition structures.

To show that $\psi^{\mu}$ is uniquely determined by the axioms, suppose that $\psi$ is a CSshare function satisfying the four axioms. Then, individual symmetry implies that $\psi_{i}\left(N, v^{0},\{N\}\right)=\frac{1}{|N|}$ for all $i \in N$. Next, consider the game $\left(N, w^{T}\right), T \subset N$, with 
$w^{T}=c^{T} u^{T}, c^{T}>0$ and $u^{T}$ the unanimity game of coalition $T$. For $i \in N \backslash T$ the null player property implies that $\psi_{i}\left(N, w^{T},\{N\}\right)=0$. Individual symmetry implies that there exists a $c^{*} \in \mathbf{R}$ such that $\psi_{i}\left(N, w^{T},\{N\}\right)=c^{*}$ for all $i \in T$. Since $\psi$ is a CS-share function it must hold that $c^{*}=\frac{1}{|T|}$, and thus $\psi\left(N, w^{T},\{N\}\right)$ is determined.

For $(N, v) \in \mathcal{G}$, recall from the preliminaries that $v+v^{-}=v^{+}$, with both $v^{-}$and $v^{+}$a nonnegative linear combination of unanimity games. Hence, $\psi\left(N, v^{+},\{N\}\right)$ and $\psi\left(N, v^{-},\{N\}\right)$ follow directly from $\mu$-additivity for one-coalition structures and the fact that we determined $\psi\left(N, c^{T} u^{T},\{N\}\right), c^{T} \geq 0$, above. Consequently the uniqueness of $\psi(N, v,\{N\})$ follows directly from applying $\mu$-additivity for one-coalition structures to $v+v^{-}=v^{+}$.

Finally, for arbitrary $(N, v, P) \in \mathcal{G P}$, uniqueness of $\psi(N, v, P)$ then follows directly from applying $\mu$-multiplication.

Q.E.D.

Instead of the coalition structure $\{N\}$ in $\mu$-additivity for one-coalition structures we could also require $\mu$-additivity just for coalition structures with $|N|$ coalitions, i.e. the coalition structures $\left\{\{i\}_{i \in N}\right\}$.

Axiom 4.6 ( $\mu$-additivity for $n$-coalition structures) Let $\mu: \mathcal{G} \rightarrow \mathbf{R}$ be given. For every $(N, v),(N, w) \in \mathcal{G}$ it holds that

$\mu(N, v+w) \psi\left(N, v+w,\left\{\{i\}_{i \in N}\right\}\right)=\mu(N, v) \psi\left(N, v,\left\{\{i\}_{i \in N}\right\}\right)+\mu(N, w) \psi\left(N, w,\left\{\{i\}_{i \in N}\right\}\right)$.

In order to characterize the class of share functions $\psi^{\mu}$ using this $\mu$-additivity of $|N|$ coalition structures we need to replace individual symmetry by coalitional symmetry stating that the total payoffs for two coalitions that are symmetric in the external game $\left(M, v^{P}\right)$ are the same.

Axiom 4.7 (Coalitional symmetry) If $P_{k}, P_{l} \in P \in \mathcal{P}^{N}$ are symmetric players in the external game $\left(M, v^{P}\right)$ induced by $(N, v, P) \in \mathcal{G P}$, then $\sum_{i \in P_{k}} \psi_{i}(N, v, P)=$ $\sum_{i \in P_{l}} \psi_{i}(N, v, P)$.

Theorem 4.8 Let $\mu: \mathcal{G} \rightarrow \mathbf{R}$ be positive, additive and symmetric on $\mathcal{G}$. Then the function $\psi^{\mu}$ is the unique $C S$-share function on $\mathcal{G}$ that satisfies $\mu$-multiplication, the null player property, coalitional symmetry and $\mu$-additivity for $|N|$-coalition structures. 


\section{PROOF}

From Theorem 4.5 it directly follows that $\psi^{\mu}$ satisfies $\mu$-multiplication and the null player property. If $P_{k}, P_{l} \in P$ are symmetric players in $\left(M, v^{P}\right)$ then symmetry of $\rho^{\mu}$, and $\rho^{\mu}$ being a share function imply that $\sum_{i \in P_{k}} \psi_{i}^{\mu}(N, v, P)=\sum_{i \in P_{k}} \rho_{i}^{\mu}\left(P_{k}, v_{\mu}^{P_{k}}\right)$. $\rho_{k}^{\mu}\left(M, v^{P}\right)=\rho_{k}^{\mu}\left(M, v^{P}\right)=\rho_{l}^{\mu}\left(M, v^{P}\right)=\sum_{i \in P_{l}} \rho_{i}^{\mu}\left(P_{l}, v_{\mu}^{P_{l}}\right) \cdot \rho_{l}^{\mu}\left(M, v^{P}\right)=\sum_{i \in P_{l}} \psi_{i}^{\mu}(N, v, P)$. This shows that $\psi^{\mu}$ satisfies coalitional symmetry. Showing that $\psi^{\mu}$ satisfies $\mu$ additivity for $n$-coalition structures follows in the same way as it is shown that $\psi^{\mu}$ satisfies $\mu$-additivity for one-coalition structures in the proof of Theorem 4.5 but using consistency property 3 of Theorem 3.1 instead of property 2. Uniqueness of $\psi^{\mu}$ follows in a similar way as in the proof of Theorem 4.5.

Q.E.D.

Finally we mention that $\mu$-multiplication also can be replaced by the similar axiom in terms of shares $\rho^{\mu}$ and adding consistency property 2 (in Theorem 4.5) or consistency property 3 (in Theorem 4.8) as stated in Theorem 3.1.

\section{An axiomatization using consistency}

In Section 3 we defined for given function $\mu$ the CS-share function $\psi^{\mu}$ by using the multiplication property and showed that this function satisfies three consistency properties. In the previous section we characterized this class of share functions using the multiplication property as one of the axioms. Next we give an axiomatization of the CS-share functions $\psi^{\mu}$ that uses consistency property 1 . As in the previous section, we state the axioms in terms of CS-shares functions. So, in the following axioms, let $\mu$ be a symmetric, additive and positive function on $\mathcal{G}$ and $\psi$ a CS-share function on $\mathcal{G P}$.

Axiom 5.1 (Consistency) If $(N, v, P) \in \mathcal{G P}$ and $P_{k} \in P$ then $\sum_{i \in P_{k}} \psi_{i}(N, v, P)=$ $\psi_{k}\left(M, v^{P},\{M\}\right)$.

Unlike $\mu$-multiplication this axiom does not depend on the $\mu$-function. Finally, we generalize $\mu$-additivity for one-coalition structures (and $\mu$-additivity for $n$-coalition structures).

Axiom 5.2 ( $\mu$-additivity for coalition structures) For $(N, v, P),(N, w, P) \in \mathcal{G P}$ let the characteristic function $z$ be given by $z=v+w$. For $i \in P_{k} \in P$ it holds that

$$
\mu\left(P_{k}, z_{\mu}^{P_{k}}\right) \mu\left(M, z^{P}\right) \psi_{i}(N, z, P)=
$$




$$
\begin{aligned}
& \left(\mu\left(P_{k}, v_{\mu}^{P_{k}}\right) \psi_{i}\left(P_{k}, v_{\mu}^{P_{k}},\left\{P_{k}\right\}\right)+\mu\left(P_{k}, w_{\mu}^{P_{k}}\right) \psi_{i}\left(P_{k}, w_{\mu}^{P_{k}},\left\{P_{k}\right\}\right)\right) \times \\
& \left(\mu\left(M, v^{P}\right) \psi_{P_{k}}\left(M, v^{P},\{M\}\right)+\mu\left(M, w^{P}\right) \psi_{P_{k}}\left(M, w^{P},\{M\}\right)\right) .
\end{aligned}
$$

When in Theorem 4.5 the axiom of $\mu$-multiplication is replaced by consistency and the axiom of $\mu$-additivity for one-coalition structures is strengthened to $\mu$-additivity for coalition structures we again obtain a characterization for the CS-share functions $\psi^{\mu}$ as defined in Theorem 3.1.

Theorem 5.3 Let $\mu: \mathcal{G} \rightarrow \mathbf{R}$ be positive, additive and symmetric on $\mathcal{G}$. Then the function $\psi^{\mu}$ is the unique CS-share function on $\mathcal{G}$ that satisfies consistency, the null player property, individual symmetry and $\mu$-additivity for coalition structures.

\section{ProOF}

Again, we first show that $\psi^{\mu}$ satisfies the four properties. From Theorem 3.1, properties 1 and 2, it follows that $\psi^{\mu}$ satisfies the consistency axiom. From Theorem 4.5 we know that $\psi^{\mu}$ satisfies the null player property and individual symmetry. Further, let $(N, v, P),(N, w, P) \in \mathcal{G P}$ and let $z=v+w$. To prove $\mu$-additivity for coalition structures we distinguish three cases.

First, suppose that $(N, z)$ is a null game. Since $z=v^{0}$ if and only if $v=w=v^{0}$, $\mu$-additivity for coalition structures follows since $z=v^{0}$ also implies that $z^{P}$ and $z_{\mu}^{P_{k}}$ are null games for all $P_{k} \in P$.

Next, suppose that $(N, z) \in \mathcal{G}^{+}$, and let $i \in P_{k} \in P$ be a null player in $(N, z)$. Then $i$ also is a null player in both $(N, v)$ and $(N, w)$, because both games are monotone games. Using similar arguments as when proving the null player property in the proof of Theorem 4.5 it follows that $\psi_{i}^{\mu}(N, z, P)=0$. Further, $\mu\left(P_{k}, v_{\mu}^{P_{k}}\right) \cdot \rho_{i}^{\mu}\left(P_{k}, v_{\mu}^{P_{k}}\right)=0$ since $i$ is a null player in $\left(P_{k}, v_{\mu}^{P_{k}}\right)$ and either $v_{\mu}^{P_{k}}=v^{0}$ (in which case $\mu\left(P_{k}, v_{\mu}^{P_{k}}\right)=0$ ) or $v_{\mu}^{P_{k}} \neq v^{0}$ (in which case $\rho_{i}^{\mu}\left(P_{k}, v_{\mu}^{P_{k}}\right)=0$ ). Similarly $\mu\left(P_{k}, w_{\mu}^{P_{k}}\right) \cdot \rho_{i}^{\mu}\left(P_{k}, w_{\mu}^{P_{k}}\right)=0$. So, also in this case $\mu$-additivity for coalition structures holds.

Finally, if $i$ is not a null player in $(N, z) \in \mathcal{G}^{+}$, then $\mu\left(P_{k}, z_{\mu}^{P_{k}}\right) \cdot \mu\left(M, z^{P}\right)>0$ and using $\mu$-additivity of $\rho^{\mu}$ we can derive that

$$
\begin{gathered}
\mu\left(P_{k}, z_{\mu}^{P_{k}}\right) \mu\left(M, z^{P}\right) \psi_{i}^{\mu}(N, z, P)=\mu\left(P_{k}, z_{\mu}^{P_{k}}\right) \mu\left(M, z^{P}\right) \rho_{i}^{\mu}\left(P_{k}, z_{\mu}^{P_{k}}\right) \rho_{k}^{\mu}\left(M, z^{P}\right) \\
=\mu\left(P_{k}, z_{\mu}^{P_{k}}\right) \mu\left(M, z^{P}\right)\left(\frac{\mu\left(P_{k}, v_{\mu}^{P_{k}}\right) \rho_{i}^{\mu}\left(P_{k}, v_{\mu}^{P_{k}}\right)+\mu\left(P_{k}, w_{\mu}^{P_{k}}\right) \rho_{i}^{\mu}\left(P_{k}, w_{\mu}^{P_{k}}\right)}{\mu\left(P_{k}, z_{\mu}^{P_{k}}\right)}\right) \times
\end{gathered}
$$




$$
\begin{array}{r}
\left(\frac{\mu\left(M, v^{P}\right) \rho_{k}^{\mu}\left(M, v^{P}\right)+\mu\left(M, w^{P}\right) \rho_{k}^{\mu}\left(M, w^{P}\right)}{\mu\left(M, z^{P}\right)}\right) \\
=\left(\mu\left(P_{k}, v_{\mu}^{P_{k}}\right) \rho_{i}^{\mu}\left(P_{k}, v_{\mu}^{P_{k}}\right)+\mu\left(P_{k}, w_{\mu}^{P_{k}}\right) \rho_{i}^{\mu}\left(P_{k}, w_{\mu}^{P_{k}}\right)\right) \times \\
\left(\mu\left(M, v^{P}\right) \rho_{k}^{\mu}\left(M, v^{P}\right)+\mu\left(M, w^{P}\right) \rho_{k}^{\mu}\left(M, w^{P}\right)\right) .
\end{array}
$$

Consistency property 2 of Theorem 3.1 then implies that $\psi^{\mu}$ also satisfies $\mu$-additivity for coalition structures in this case.

To show uniqueness, suppose that $\psi$ satisfies the four axioms. For a null game, $\psi\left(N, v^{0}, P\right)$ is uniquely determined by individual symmetry. Next, consider for $T \subset$ $N$, the scaled unanimity game $\left(N, w^{T}\right)$, with $w^{T}=c^{T} u^{T}, c^{T}>0$, and let $P=$ $\left\{P_{1}, \ldots, P_{m}\right\}$ be a coalition structure in $\mathcal{P}^{N}$. For $i \in N \backslash T$ the null player property implies that $\psi_{i}\left(N, w^{T}, P\right)=0$. To obtain $\psi_{i}\left(N, w^{T}, P\right)$ for $i \in T$, first define $K(T, P):=\left\{k \in M \mid P_{k} \cap T \neq \emptyset\right\}$. Then, all coalitions $P_{k} \in P$ such that $k \in K(T, P)$ are symmetric in the external game $\left(M,\left(w^{T}\right)^{P}\right)$. Individual symmetry then implies that there exists a $c^{*} \in \mathbf{R}$ such that $\psi_{k}\left(M,\left(w^{T}\right)^{P},\{M\}\right)=c^{*}$ for all $k \in K(T, P)$. By $\psi$ being a CS-share function and $\sum_{i \in P_{k}} \psi_{i}\left(N, w^{T}, P\right)=0$ for all $k \in M \backslash K(T, P)$, we have that $\psi_{k}\left(M,\left(w^{T}\right)^{P},\{M\}\right)=c^{*}=\frac{1}{|K(T, P)|}$ for all $k \in K(T, P)$. Consistency then implies that $\sum_{i \in P_{k}} \psi_{i}\left(N, w^{T}, P\right)=\frac{1}{|K(T, P)|}$ for $k \in K(T, P)$. Since all $i \in P_{k} \cap T$ are symmetric players in $\left(N, w^{T}\right)$, and $\psi_{i}\left(N, w^{T}, P\right)=0$ for $i \in N \backslash T$, individual symmetry implies that $\psi_{i}\left(N, w^{T}, P\right)=\frac{1}{\left|P_{k} \cap T\right| \cdot|K(T, P)|}$ for $i \in P_{k} \cap T$ and thus $\psi\left(N, w^{T}, P\right)$ is uniquely determined for any $w^{T}$ and coalition structure $P$. Finally for arbitrary $(N, v) \in \mathcal{G}$ and coalition structure $P, \psi\left(N, v^{+}, P\right)$ and $\psi\left(N, v^{-}, P\right)$ follow directly from $\mu$-additivity for coalition structures and consequently the uniqueness of $\psi(N, v, P)$ follows directly from applying $\mu$-additivity for coalition structures to $v+v^{-}=v^{+}$.

Q.E.D.

We can restate $\mu$-additivity for coalition structures using coalition structures $\left\{\{i\}_{i \in P_{k}}\right\}$ and $\left\{\{k\}_{k \in M}\right\}$ instead of coalition structures $\left\{P_{k}\right\}$, respectively, $\{M\}$. To characterize the share functions $\psi^{\mu}$ we then also have to replace individual symmetry by coalitional symmetry as done in going from Theorem 4.5 to Theorem 4.8. Finally, we can replace consistency in Theorem 5.3 by coalitional symmetry. That gives a characterization using both individual and coalitional symmetry. 


\section{Conclusion}

In this paper we discussed a general approach to defining CS-share functions for games in coalition structure using a multiplication property. We showed that all these CSshare functions are consistent in the sense that the total payoff of all players in an a priori coalition is equal to the payoff of this coalition in the external game between the coalitions. We gave axiomatic characterizations using these multiplication and consistency properties.

Two special cases are the Shapley- and Banzhaf CS-share functions. The Shapley CS-share function is obtained by normalizing the Owen CS-value function to one. However, the Banzhaf CS-share function discussed in this paper is different from the Banzhaf type CS-share function that is obtained a la Owen (1981)'s Banzhaf CSvalue function. In the Banzhaf value each marginal contribution has an equal weight. Generalizing this to games in coalition structure, Owen (1981) assigns equal weights to each marginal contribution of a coalition within the coalition structure and within such a coalition to each marginal contribution of the players within that coalition. Replacing in equation (1) the Shapley weights by Banzhaf weights yields the value function $\theta$ on $\mathcal{G P}$ defined by

$$
\theta_{i}(N, v, P)=\sum_{\substack{L \subset M \\ L \not \supset k}} \sum_{\substack{E \subset P_{k} \\ E \ni i}} 2^{-(m-1)} \cdot 2^{-\left(\left|P_{k}\right|-1\right)}(v(E \cup P(L))-v((E \backslash\{i\}) \cup P(L))),
$$

for all $i \in P_{k}, k \in M$. The corresponding share function $\psi$ which, for every $i \in N$ and $(N, v) \in \mathcal{G}^{+}$, is given by $\psi_{i}(N, v, P)=\frac{\theta_{i}(N, v, P)}{\sum_{j \in N} \theta_{j}(N, v, P)}$, is not the same as the Banzhaf CS-share function discussed in this paper. In particular, it does not satisfy the multiplication property nor consistency.

\section{References}

[1] Aumann, R.J., Drèze J.H.: Cooperative Games with Coalition Structure. International Journal of Game Theory. 3 (1974) 217-237.

[2] Banzhaf, J.F.: Weighted Voting Doesn't Work: A Mathematical Analysis. Rutgers Law Review. 19 (1965) 317-343.

[3] Brink, R. van den, Laan, G. van der: Axiomatizations of the Normalized Banzhaf Value and the Shapley Value. Social Choice and Welfare. 15 (1998) 567-582. 
[4] Brink, R. van den, Laan, G. van der: The Normalized Banzhaf Value and the Banzhaf Share Function. In: Game Theory and Applications Vol. 4 (eds. V. Mazalov and L. Petrosjan). Nova Science Publishers, New York (1999a) pp 1131.

[5] Brink, R. van den, Laan G. van der: Core Concepts for Share Vectors. CentER and TI Discussion Paper, to appear in Social Choice and Welfare (1999b).

[6] Deegan, J., Packel, E.W.: A New Index of Power for Simples n-Person Games. International Journal of Game Theory. 7 (1979) 113-123.

[7] Dubey, P., Shapley, L.S.: Mathematical Properties of the Banzhaf Power Index. Mathematics of Operations Research. 4 (1979) 99-131.

[8] Grabisch, M., Roubens, M.: An Axiomatic Approach to the Concept of Interaction among Players in Cooperative Games. International Journal of Game Theory. 28 (1999) 547-565.

[9] Haller, H.: Collusion Properties of Values. International Journal of Game Theory. 23 (1994) 261-281.

[10] Harsanyi, J.C.: A Bargaining Model for Cooperative $n$-Person Games, in: Contributions to the Theory of Games IV (eds. A.W. Tucker and R.D. Luce). Princeton University Press, Princeton NJ, (1959) pp 325-355.

[11] Harsanyi, J.C.: A Simplified Bargaining Model for the $n$-Person Game. International Economic Review. 4 (1963) 194-220.

[12] Hart S., Kurz M.: Endogeneous Formation of Coalitions. Econometrica. 51 (1983) 1047-1064.

[13] Laan, G. van der, Brink, R. van den: Axiomatization of a Class of Share Functions for N-Person Games. Theory and Decision. 44 (1998) 117-148.

[14] Laan, G. van der, Brink, R. van den: A Banzhaf Share Function for Cooperative Games in Coalition Structure. Center and TI Discussion Paper (1999).

[15] Nowak, A.S.: An the Axiomatization of the Banzhaf Value without the Additivity Axiom. International Journal of Game Theory. 26 (1997) 137-141. 
[16] Lehrer, E.: An Axiomatization of the Banzhaf Value. International Journal of Game Theory. 17 (1988) 89-99.

[17] Owen G.: Multilinear Extensions and the Banzhaf Value. Naval Research Logistics Quarterly. 22 (1975) 741-750.

[18] Owen G.: Values of Games with a Priori Unions. Essays in Mathematical Economics and Game Theory (eds. R. Henn and O. Moeschlin). Springer Verlag, Berlin (1977) pp 76-88.

[19] Owen, G.: Modification of the Banzhaf-Coleman Index for Games with a Priori Unions. Power, Voting and Voting Power (ed. M.J. Holler). Physica-Verlag, Wurzburg (1981) 232-238.

[20] Shapley, L.S.: A Value for $n$-Person Games. Annals of Mathematics Studies 28 (Contributions to the Theory of Games Vol.2) (eds. H.W. Kuhn and A.W. Tucker). Princeton University Press, Princeton (1953) pp 307-317.

[21] Winter E.: A Value for Cooperative Games with Levels Structure of Cooperation. International Journal of Game Theory. 18 (1989) 227-240. 